\title{
Effect of metal ion doping on the photocatalytic activity of alumino- phosphates
}

\author{
AVIJIT KUMAR PAUL, MANIKANDA PRABU, GIRIDHAR MADRAS* and \\ SRINIVASAN NATARAJAN* \\ Solid State and Structural Chemistry Unit, Indian Institute of Science, Bangalore 560012 \\ e-mail: giridhar@ $@$ chemeng.issc.ernet.in; snatarajan $@$ sscu.iisc.ernet.in
}

\begin{abstract}
The metal ions $\left(\mathrm{Ti}^{+4}, \mathrm{Mg}^{+2}, \mathrm{Zn}^{+2}\right.$ and $\mathrm{Co}^{+2}$ ) have been substituted in place of $\mathrm{Al}^{+3}$ in aluminophosphates (AlPOs). These compounds were used for the first time as possible photocatalysts for the degradation of organic dyes. Among the doped AlPOs, ZnAlPO-5, CoAlPO-5, MgAlPO-11, 18 and 36 did not show any photocatalytic activity. MgAlPO-5 showed photocatalytic activity and different loading of $\mathrm{Mg}(4,8,12$ atom \% of $\mathrm{Mg}$ ) were investigated. The activity can be enhanced by the increasing of concentration of the doped metal ions. TiAlPO-5 $(4,8,12$ atom \% of Ti) showed the highest photocatalytic activity among all the compounds and its activity was compared to that of Degussa P25 $\left(\mathrm{TiO}_{2}\right)$. The activity of photocatalysts was correlated with the diffuse reflectance and photoluminescence spectra.
\end{abstract}

Keywords. Aluminophosphates; doping; photocatalysis; dye degradation.

\section{Introduction}

Microporous aluminosilicates have commanded attention not solely due to the Bronsted acidic catalytic properties, but also for the entire sweep of Lewis acidity based catalytic properties and redox activity that lead to interesting chemical reactions to both the academic chemist as well as to the industrialist. ${ }^{1-4}$ The discovery of aluminophosphates (AlPO$\mathrm{n}, \mathrm{n}$ corresponds to mathematical number) in 1982 by Flanigen and co-workers ${ }^{5}$ with zeolite-like structures provided another family of compounds with open framework structures. AlPOs are intrinsically more polar than aluminosilicates and it is easier to substitute $\mathrm{Al}^{+3}$ site in the tetrahedral framework with a wide range of metal ions. This leads to a family of structures known as metal (Me)-substituted AlPOs (Me-AlPOs). ${ }^{6}$ The heterogeneous catalytic properties of Me-AlPOs have been studied extensively during the last decade. Thomas and co-workers have established that substitutions of bivalent metal ions in AlPOs give rise to heterogeneous catalysts, especially when the substituted atom is a transition element. $^{7,8}$

The use of visible or ultra-violet (UV) light for carrying out photo-assisted chemical reactions has attracted the attention of scientists. Photocatalytic studies of many organic reactions have been carried

*For correspondence out in the presence of aluminosilicates, notably zeolite-Y (faujasite) ${ }^{9,10}$ and also on many titaniumcontaining compounds. ${ }^{11-13}$ The photocatalytic activity under visible light has been studied using the compounds based on titanate phases and other nanoparticles. ${ }^{14-16}$ The single site photocatalytic studies on $\mathrm{TiO}_{2}$ and other related compounds have been reviewed recently. ${ }^{17}$ Since the AlPOs have structures that are comparable to the zeolites, it would be interesting to study the photo-assisted reactions employing AlPOs as the photocatalysts. A careful search of the literature reveals that such studies have not been attempted in any of the microporous AlPOs $(<20 \AA$ pore diameter), though some effort has been expended for such a study employing mesoporous aluminophosphates $(20-500 \AA$ pore diameter) and related compounds. ${ }^{18}$

We have been investigating the use of UV-visible light for the degradation of organics in the presence of a suitable catalyst. ${ }^{19-21}$ The degradation of organics is important, especially in the treatment of wastewater from industries. Of the many processes that are known for the degradation of organics, the wet air oxidation (WAO) and advanced oxidation processes (AOP) using Fenton's reagent and photochemical reactions in the presence of hydrogen peroxide $^{22}$ are important. The WAO process requires elevated temperatures $\left(150-325^{\circ} \mathrm{C}\right)$ and high oxygen pressures, which lead to higher energy costs. The advanced photochemical process, on the other hand, 
produces a large number of intermediates, some of which could be toxic. The photocatalytic degradation is, thus, advantageous over these other processes as it reduces the waste disposal issues.

An ideal photocatalyst would utilize visible and/or near UV light, be biologically and chemically inert, photostable (not prone to photocorrosion), inexpensive and non-toxic. Of the many materials that have been investigated, $\mathrm{TiO}_{2}$ and related oxides appear to have many of these qualities and were studied extensively. ${ }^{11-17}$ Recently, metal-organic frameworks (MOFs) with $\pi$-donors have also been shown to be active for the photodegradation of dyes and organics. ${ }^{23-25}$ In the continued research aimed at finding new materials as photocatalysts, we have been interested in AlPOs. A report in Chinese ${ }^{26}$ has discussed the degradation of methyl orange with Fe-doped AlPOs. However, a detailed study on various metal-doped AlPOs for photocatalysis is not available. In this present study, MeAlPOs have been examined as photocatalysts for the degradation of a variety of organic dyes; xanthene (Rhodamine blue, $\mathrm{RBL}$ ), anthraquinoic (Alizarin green, $\mathrm{AG}$ ), azoic (Orange G, OG), heteropolyatomic (Methylene blue, $\mathrm{MB}$ ), and ionic (trans-4-[4-(dimethylamino)styryl]1-methylpyridiniumiodide, DSMP) and the results are presented here. The effects of doped metal ions have also been studied for the photocatalytic activity.

\section{Experimental}

\subsection{Materials}

The organic dyes and other compounds for the photocatalytic experiments, $\mathrm{MB}, \mathrm{RBL}, \mathrm{OG}, \mathrm{AG}$, and DSMP (S.D. Fine-Chem. Ltd, India), were used as received. The water used was double distilled filtered through a Millipore membrane. All chemicals were purchased from Aldrich Chemicals for the preparation of AlPOs.

\subsection{Synthesis and initial characterization}

All the compounds investigated in the present study were prepared employing the well-established synthesis procedures. ${ }^{27}$ In a typical experiment for the preparation of $\mathrm{AlPO}-5$, the reagents, $\mathrm{Al}(\mathrm{O}-\mathrm{iPr})_{3}$, $\mathrm{H}_{3} \mathrm{PO}_{4}(85 \mathrm{wt} \%), \mathrm{Et}_{3} \mathrm{~N}$ and $\mathrm{H}_{2} \mathrm{O}$ were taken in the mole ratio, $1: 1: 1.5: 222$. The aluminium precursor,
$\mathrm{Al}(\mathrm{O}-\mathrm{iPr})_{3}$ (LEO-Chemica, 99\%) was taken in $4 \mathrm{ml}$ water based on the above molar ratio and then $\mathrm{H}_{3} \mathrm{PO}_{4}$ (Merck, $85 \mathrm{wt} \%$ ) was added drop-wise. The organic amine $\left(\mathrm{Et}_{3} \mathrm{~N}\right.$; Aldrich, 99.5\%) was added after stirring the mixture for half an hour. Finally, $0.5 \mathrm{ml}$ of HF (Rankem, $40 \mathrm{wt} \%$ in water) was added to the mixture and stirred for one hour. The mixture was heated in a PTFE- lined stainless steel autoclave at $180^{\circ} \mathrm{C}$ for $36 \mathrm{~h}$. The resulting white coloured solid was vacuum filtered, washed with deionized water and dried under atmospheric conditions. A similar synthesis procedure was adopted for the preparation of the metal substituted AlPO-5 ( $\mathrm{Me}=\mathrm{Co}, \mathrm{Zn}, \mathrm{Mg}$ and $\mathrm{Ti})$. The metal acetate salts $(\mathrm{CDH}, \mathrm{AR}$ grade) were taken for $\mathrm{Co}, \mathrm{Zn}, \mathrm{Mg}$ and titanium-isopropoxide was taken for Ti doped AlPOs. The molar concentration of the salts were taken as $\mathrm{x} \%$ (varied from $4 \%$ to $12 \%$ ) to obtain the product $\mathrm{Me}_{x} \mathrm{Al}_{(1-x)} \mathrm{PO}_{4}$ where $x$ is atom $\%$. The metal salts were added before the amine and HF were added. In all the cases, white powder samples were obtained, except CoAlPO which was blue in colour.

The Ti and Mg doped ( 8 atom \%) AlPO- $n(n=11$, 18 and 36) were also prepared employing standard synthesis procedures available in the literature. ${ }^{27}$ The different MeAlPO- $n$ was synthesized by using different organic amines as a template. The $\mathrm{pH}$ of the reaction mixture has an important role in the synthesis of MeAlPO- $n$. The MeAlPO-11 was synthesized by using the dipropylamine (Aldrich, 99\%) instead of $\mathrm{Et}_{3} \mathrm{~N}$ and $\mathrm{HF}$ mixture. The $\mathrm{pH}$ of the reaction mixture was 6 and heated in a PTFE-lined stainless steel autoclave at $200^{\circ} \mathrm{C}$ for $24 \mathrm{~h}$. The MeAlPO-18 was synthesized by using $N, N$ diisopropylethylamine (Aldrich, 99\%) and the $\mathrm{pH}$ of the reaction mixture was 8 . The product was obtained from the heating of the mixture at $135^{\circ} \mathrm{C}$ for $96 \mathrm{~h}$. The MeAlPO-36 was synthesized by using tripropylamine (Aldrich, 98\%) and the $\mathrm{pH}$ of the reaction mixture was 7 . The reaction mixture was heated at $125^{\circ} \mathrm{C}$ for $24 \mathrm{~h}$ and $150^{\circ} \mathrm{C}$ for $24 \mathrm{~h}$. In all the cases, the products MeAlPO- $n(n=11,18$ and 36) were white. The synthesized samples were heated at $550^{\circ} \mathrm{C}$ for $12 \mathrm{~h}$ in a flow of oxygen to remove the occluded organic template molecules and to yield a microporous samples.

All the compounds were characterized by a variety of techniques that include powder X-ray diffraction (XRD), FTIR, diffuse reflectance spectroscope, photoluminescence spectroscope, SEM and BET surface area. The powder XRD patterns were 
recorded on the samples in the $2 \theta$ range $5-50^{\circ}$ using $\mathrm{Cu}-\mathrm{K} \alpha$ radiation (Philips, X'pert-Pro, $\lambda=1.5418 \AA$ ). Infrared (IR) spectroscopic studies have been carriedout in the mid-IR region (4000 to $400 \mathrm{~cm}^{-1}$ ) on $\mathrm{KBr}$ pellets (Perkin Elmer, SPECTRUM 1000). The diffuse reflectance spectra of all the samples (as prepared and calcined) were recorded using UVvisible spectrophotometer (Perkin-Elmer model Lambda 35). The Kubelka-Munk $\left[F(R)=(1-R)^{2} /\right.$ $2 R]$ transformations were carried out on the UV-Vis reflectance data to obtain an absorbance like spectra. XAFS of the TiAlPO-5 have been done with Ti-HMS as a reference of tetrahedral environment. The ICP analysis of AlPO-5, MgAlPO-5 (4, 8 and 12 at \%) and TiAlPO-5 (4, 8 and 12 at\%) was carried out in Optical Emission Spectrometer (PerkinElmer; Optima 2100DV). For ICP analysis, the standard solution for the calibration of $\mathrm{Ti}$ was prepared by dissolving titanium-isopropoxide in water. Similarly, the standard solutions for $\mathrm{Al}, \mathrm{Mg}$ and $\mathrm{P}$ were prepared by dissolving nitrate salts of $\mathrm{Al}, \mathrm{Mg}$ and $\mathrm{H}_{3} \mathrm{PO}_{4}$ in water. The AlPO-5 and MeAlPO-5 samples were digested in $\mathrm{HNO}_{3}$ and $\mathrm{HCl}$ mixture and the dilute solution was prepared in water. The amount of $\mathrm{Ti}, \mathrm{Mg}, \mathrm{Al}$ and $\mathrm{P}$ were determined from the calibrations. To ensure accuracy, several samples of AlPOs were digested and analysed in ICP. The photoluminescence emission spectra were recorded in Luminescence spectrometer (PerkinElmer model LS 55). SEM studies have been carried out on FEI-SEM (QUANTA). The surface area of the materials was determined by volumetric method using $\mathrm{N}_{2}$ as the adsorbent (Belsorp, Japan).

\subsection{Degradation experiments}

The photochemical reactor employed in this study is similar to that employed earlier in our studies. ${ }^{23-25}$ The mercury vapor lamp radiated predominantly at $365 \mathrm{~nm}$ corresponding to the energy of $3.4 \mathrm{eV}$. The photon flux was measured by conducting actinometric experiments with $o$-nitrobenzaldehyde and the flux was $2.6 \times 10^{-6} \mathrm{~mol} / \mathrm{m}^{2} \mathrm{~s}$. For the photocatalytic degradation studies, the organic dyes were dissolved in double distilled Millipore filtered water. All the experiments were carried out at the natural $\mathrm{pH}$ of the solution. The $\mathrm{pH}$ of the solutions was between 6 and 7 and did not vary during the photocatalytic reactions. The reactions were carried out at $30^{\circ} \mathrm{C}$, which was maintained by circulating water in annulus of the jacketed quartz reactor. All the compounds were stirred in dark for $30 \mathrm{~min}$ at room temperature prior to UV irradiation to ensure that the equilibrium adsorption/desorption of the substrate on the catalyst was attained. This would eliminate the possible effects of adsorption during photocatalytic reactions. However, no adsorption of the dye on the material was observed even after $12 \mathrm{~h}$ of stirring in the dark. Samples were collected at regular intervals, filtered through Millipore membrane filters, and centrifuged to remove the catalyst particles prior to the analysis.

Samples were analysed using a UV-visible spectrophotometer. The calibration for RBL, MB, AG, OG, and DSMP was based on Beer-Lambert law at its maximum absorption wavelength. ${ }^{19-22}$ Control experiments with either only UV or in the presence of AlPO-5 were carried out, which showed no degradation of the dye. The powder XRD patterns, recorded after photocatalytic reactions, did not reveal any changes indicating the phase stability of the catalysts. Experiments were conducted in triplicate for all cases and the error in the determination of the degradation rate coefficient is less than $5 \%$. For comparison, the photocatalytic performance of the commercial $\mathrm{TiO}_{2}$ (Degussa $\mathrm{P} 25,30 \mathrm{~nm}$ size, $50 \mathrm{~m}^{2} / \mathrm{g}$ surface area) was also carried out under the same experimental conditions.

\section{Results and discussion}

The X-ray powder diffraction patterns for the as prepared AlPO-5 and Me-AlPO-5 $(\mathrm{Me}=\mathrm{Co}$ and $\mathrm{Zn}$ 4 atom \%; Ti and $\mathrm{Mg}=4,8$ and 12 atom \%) were identical and compared well with that reported in the literature, indicating phase purity of the samples (see supplementary information, figures S1-S3 and S7). The XRD patterns of the Mg and Ti-AlPO- $n$ $(n=11,18$ and $36 ; 8$ atom \%) also showed that they are obtained in a phase pure form (see supplementary information, figures S4-S6 and S8-S10). The calcined samples in all the cases showed that they retain the crystallinity as well as the phase purity (see supplementary information, figures S1-S10). The PXRD pattern (figure 1) of the bulk sample of MgAlPO-5 and TiAlPO-5 (8 atom \%) has shown the pure and crystalline phase of the compounds. This has been used for the detailed photocatalytic reactions.

IR spectroscopic studies of calcined AlPO-5, MgAlPO-5 and TiAlPO-5 indicated an identical spectra in the mid IR (4000-400 $\mathrm{cm}^{-1}$ ) region (figure 2 ). All the compounds have two sharp peaks at 
about 1100 and $720 \mathrm{~cm}^{-1}$ region which can be assigned for $\mathrm{P}-\mathrm{O}$ stretching vibration of $\left(\mathrm{PO}_{4}\right)^{3-}$ and stretching vibration of $\mathrm{Al}-\mathrm{O}$ in combination with

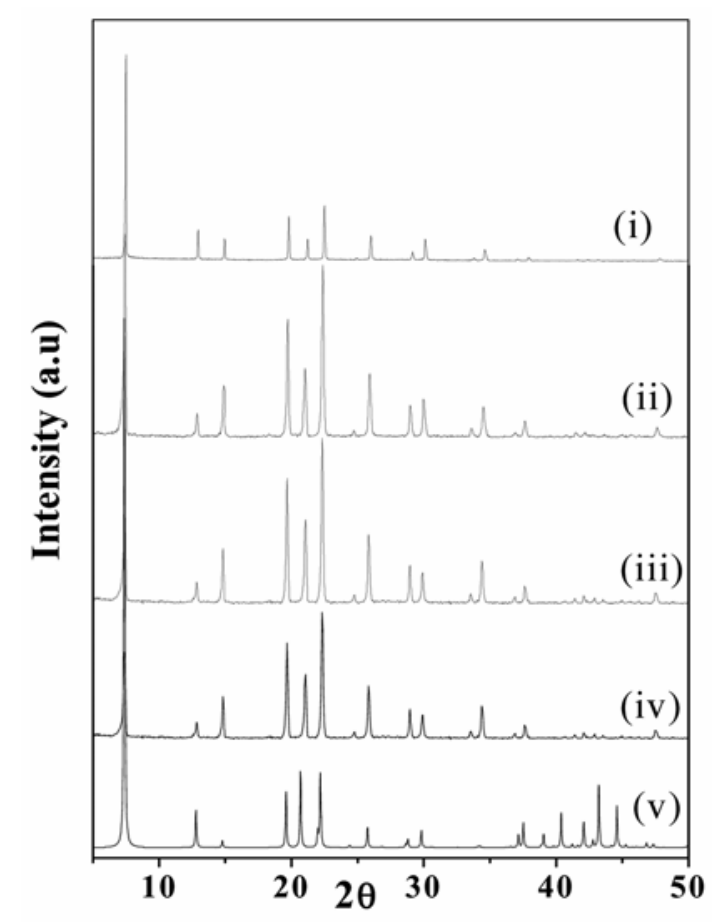

Figure 1. Powder X-ray diffraction pattern $(\mathrm{CuK} \alpha)$ for MeAlPO-5 (8\% doped): (i) as prepared MgAlPO-5, (ii) calcined MgAlPO-5, (iii) as prepared TiAlPO-5, (iv) calcined TiAlPO-5 and (v) simulated (ref. 27) MeAlPO-5.

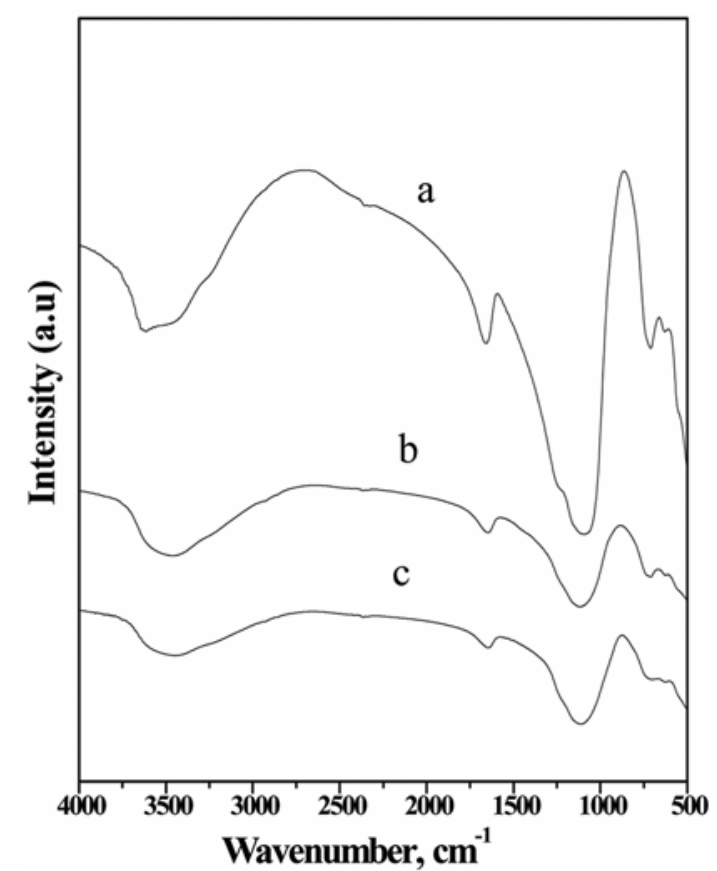

Figure 2. FTIR spectra of (a) TiAlPO-5, (b) MgAlPO-5 and (c) AlPO-5.
$\mathrm{P}-\mathrm{O}$, respectively. The other two peaks at about 3470 and $1645 \mathrm{~cm}^{-1}$ region can be assigned for the absorbed moisture and some oxidized carbonate phase of the calcined samples. One extra peak observed at $3615 \mathrm{~cm}^{-1}$ for TiAlPO-5 can be assigned to the $-\mathrm{OH}$ group, which has been discussed in detail later.

The absorbance like spectra, plotted on the calcined samples in the range of 600-200 nm indicated a peak at $\sim 267 \mathrm{~nm}$ (figure 3a). As can be noted, in the case of MgAlPO-5 (4 atom \%), an additional peak at $290 \mathrm{~nm}$ of very less intensity was also observed. The corresponding band gap values calculated from the absorbance edge would be $4.6 \mathrm{eV}$ and $4.16 \mathrm{eV}$, respectively. The higher doping of $\mathrm{Mg}$ in AlPO-5 (8 and 12 atom \%) indicates an additional peak at $305 \mathrm{~nm}$ (figure $3 \mathrm{~b}$ ). The UV-visible spectra of 8 atom \% doped MgAlPO- $n(n=5,11,18$ and 36) are shown in figure $4 \mathrm{a}$. The spectra of MgAlPO11,18 and 36 samples do not show any additional peaks other than the primary peak observed at $267 \mathrm{~nm}$, except for MgAlPO-36, which exhibit a small peak centered at $\sim 315 \mathrm{~nm}$. Since AlPO-5 and AlPO-36 have similar connectivities and comparable structure, ${ }^{27}$ the appearance of the second peak at $\sim 315 \mathrm{~nm}$ in the 8 atom $\% \mathrm{Mg}$ doped samples may be related to the distortion of structure or to the creation of a unique defect site.

The Kubelka-Munk absorbance like spectra of the TiAlPO- $n(n=5,11,18$ and 36) samples exhibit a broad peak at $260 \mathrm{~nm}$ with an additional peak at $290 \mathrm{~nm}$ (see figure 4b). The peak at $\sim 290 \mathrm{~nm}$ appears to show a shift from 290 to $305 \mathrm{~nm}$ for 4,8 and 12 atom \% TiAlPO-5 (figure 3c). A similar absorption edge due to the tetrahedral coordination of $\mathrm{Ti}$ has been observed for mesoporous titanium phosphate materials. ${ }^{28}$ Similar to this study, a shift in the absorption band to higher wavelength with increasing $\mathrm{Ti}$ loading has been observed for titanium-phosphorous mixed oxides. ${ }^{28}$ The absence of any shoulder at 330-350 $\mathrm{nm}$ in the spectra shows the absence of extra framework anatase like titanium oxide ${ }^{29}$ in which Ti is in octahedral coordination.

The band gap of the material calculated from the absorbance edge corresponds to $\sim 3 \cdot 18 \mathrm{eV}$. This observation clearly indicates that the band gap of TiAlPO-5 is less than that of MgAlPO-5. There is a marked difference in the absorbance like spectra of TiAlPO- $n$ compared to other metal substituted AlPO- $n$, as clearly observed in figure $3 \mathrm{a}$. This is due to the substitution of $\mathrm{Ti}$ in both the $\mathrm{Al}$ and $\mathrm{P}$ sites in case of TiAlPO- $n$ while the substitution of other 

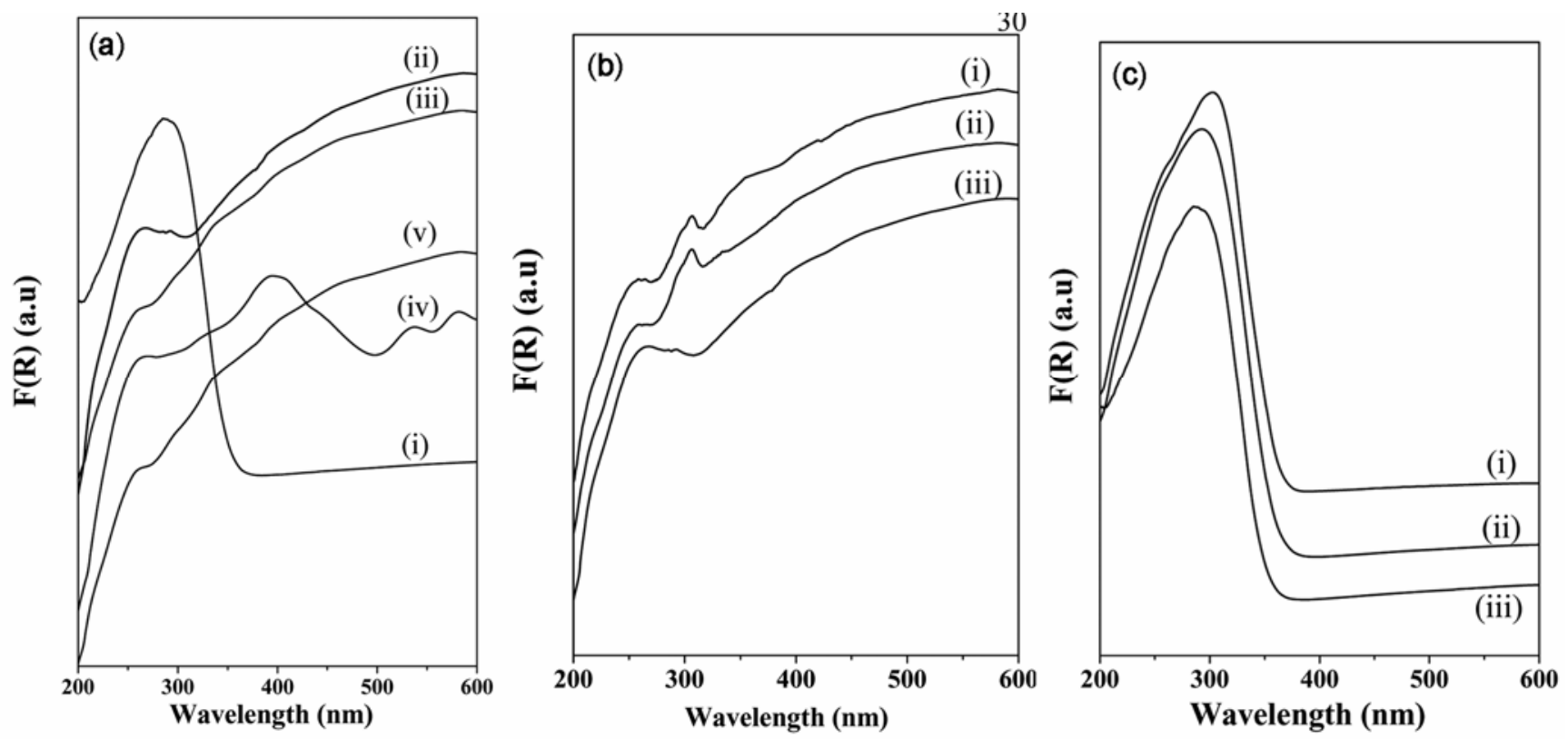

Figure 3. UV-visible Kubelka-Munk spectra of (a) (i) TiAlPO-5, (ii) MgAlPO-5, (iii) AlPO-5, (iv) CoAlPO-5 and (v) ZnAlPO-5 (4 atom \% doped Me). (b) (i) $12 \%$, (ii) $8 \%$ and (iii) $4 \%$ doped Mg in MgAlPO-5. (c) (i) $12 \%$, (ii) $8 \%$ and (iii) $4 \%$ doped $\mathrm{Ti}$ in TiAlPO-5.
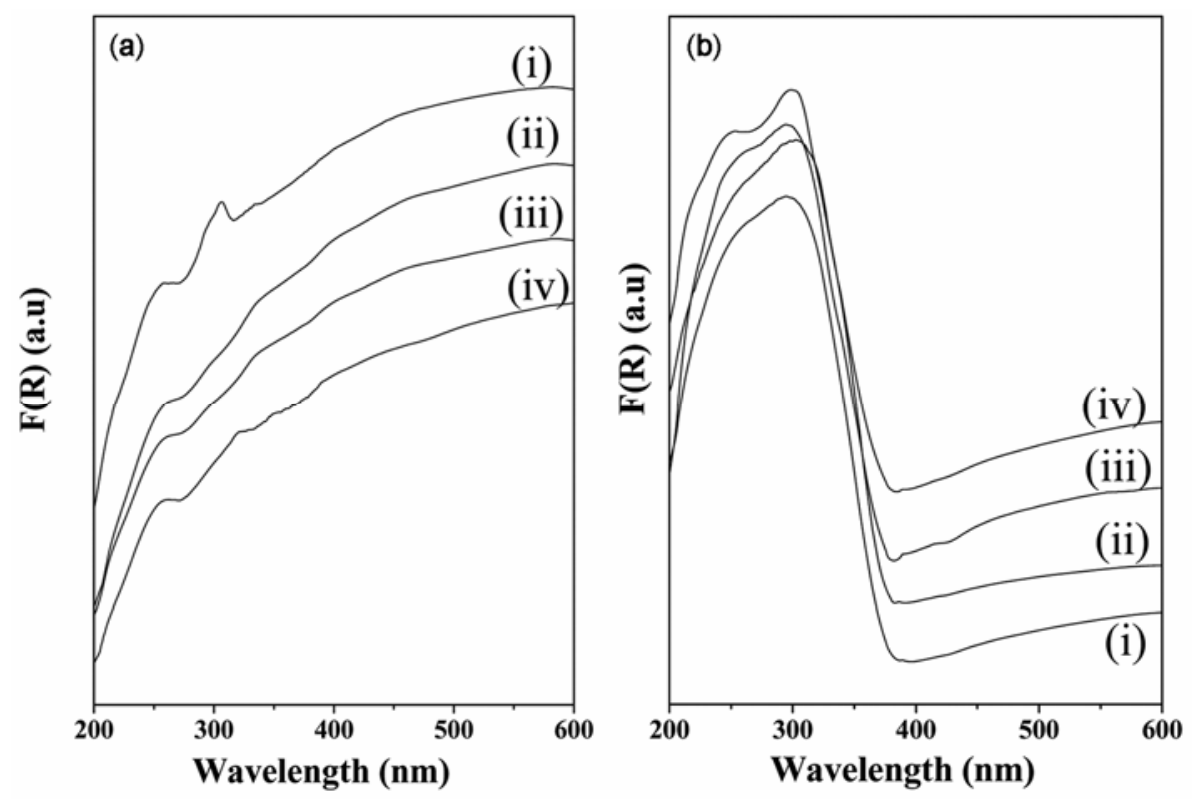

Figure 4. UV-visible absorbance like spectra of (a) (i) $\mathrm{MgAlPO}-5$, (ii) MgAlPO-11, (iii) MgAlPO-18 and (iv) MgAlPO-36. (b) (i) TiAlPO-5, (ii) TiAlPO-11, (iii) TiAlPO-18 and (iv) TiAlPO-36.

metals occurs only in the $\mathrm{Al}$ site of the other metal AlPO- $n$. This will be discussed in detail later.

The calcined samples, AlPO-5 and MeAlPO-5 $(\mathrm{Me}=\mathrm{Mg}, \mathrm{Zn}, \mathrm{Co}$; 4 atom \%), were employed in the photocatalytic degradation of methylene blue (MB; $\lambda_{\max }=660 \mathrm{~nm}$ ) (figure 5). The catalytic experiments were conducted by varying the catalyst concentra- tion. The rate coefficients do not change after $1 \mathrm{~g} / \mathrm{L}$ of the catalysts. So, the experiments were conducted with $2 \mathrm{~g} / \mathrm{L}$ of the catalysts. The plot indicates that the degradation of MB is not significant in the presence of $2 \mathrm{~g} / \mathrm{L}$ of $\mathrm{Zn}$ and Co doped AlPO-5. However, the $\mathrm{Mg}$ doped AlPO-5 shows reasonable activity. This indicates that the peak centered at 
$\sim 290 \mathrm{~nm}$, observed in the absorbance like spectra, assumes significance in determining the photocatalytic activity. If this assumption is reasonable, one could expect to see an increased photocatalytic activity in samples containing higher concentration of $\mathrm{Mg}$. Thus, we investigated the photocatalytic activity of 8 and 12 atom $\% \mathrm{Mg}$-AlPO-5 in the degradation of the organic dyes (figure 6a). As presumed, the degradation of MB appears to show some degree of dependence on the dopant concentration of $\mathrm{Mg}$ in AlPO-5. The degradation rate of MB in presence of $2 \mathrm{~g} / \mathrm{L} \mathrm{MgAlPO}-5$ is only comparable with $0 \cdot 2 \mathrm{~g} / \mathrm{L}$ of Degussa P25 (the activity, however, is much less compared to $2 \mathrm{~g} / \mathrm{L}$ Degussa P25). Since, we had observed the presence of the peak in the absorbance like spectra of the Ti doped AlPO-5 $(\sim 300 \mathrm{~nm})$, it is to be expected that these samples are likely to be active as photocatalysts. To this end, we have subjected that 4,8 and 12 atom \% doped TiAlPO-5 for the photocatalytic dissociation of MB under identical conditions (figure $6 \mathrm{~b}$ ). As can be noted, the degradation of MB in the presence of TiAlPO-5 (12\% doped) appears to be comparable with $2 \mathrm{~g} / \mathrm{L}$ Degussa P25 $\left(\mathrm{TiO}_{2}\right)$ photocatalyst after $90 \mathrm{~min}$ of degradation. The initial rate of degradation is higher in case of Degussa P25. The photocatalytic activity

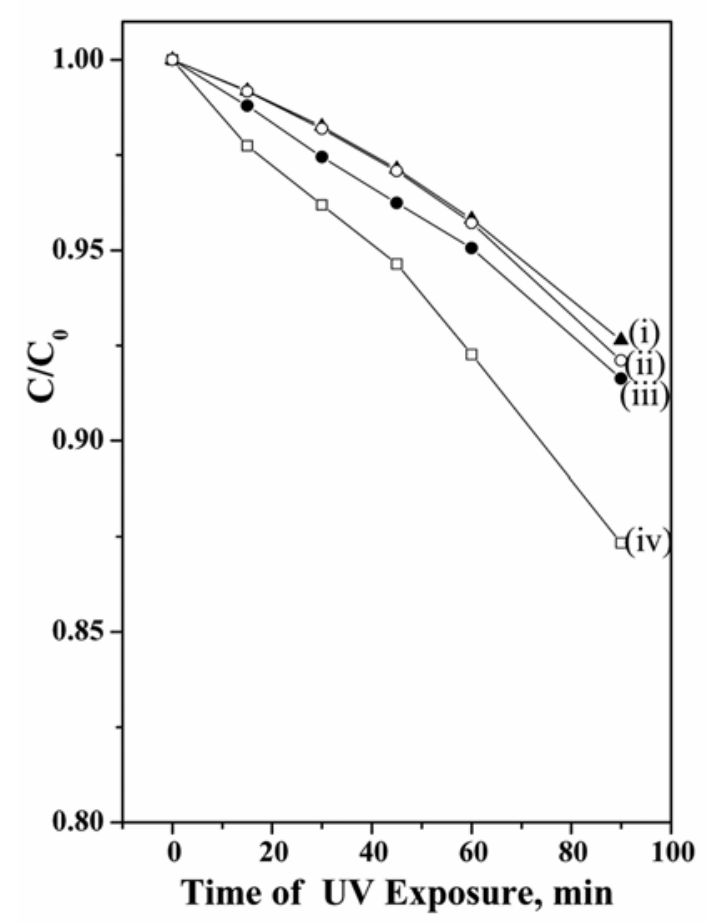

Figure 5. Degradation profiles of $25 \mathrm{ppm} \mathrm{MB}$ in presence of (i) AlPO-5, (ii) CoAlPO-5, (iii) ZnAlPO-5 and (iv) $\mathrm{MgAlPO}-5$ ( $\mathrm{Co}, \mathrm{Zn}$ and $\mathrm{Mg}=4$ atom \%). of 8 atom $\%$ and 12 atom $\%$ are more or less similar. The degradation rate increases with the increase in the dopant concentration of $\mathrm{Ti}$, but the activity appears to saturate with 8 atom \% doping. Thus, we have chosen 8 atom $\%$ Ti doped sample for further detailed investigation.

Since the Ti and Mg-AlPO-5 (8 atom \%) exhibited some degree of activity for the photocatalytic degradation of MB, the other $\mathrm{Ti}$ and Mg-doped aluminophosphate (MeAlPO- $n$ ) samples were investigated under similar conditions. The results of the study are shown in figures $7 \mathrm{a}$ and $\mathrm{b}$. We have also carried out the degradation studies as a function of varying initial concentration of $\mathrm{MB}(50,25,15$ and $10 \mathrm{ppm}$ solution of MB; $\lambda_{\max }=660$ ) using $2 \mathrm{~g} / \mathrm{L} \mathrm{Mg}$ and $\mathrm{Ti}$ doped AlPO-5, 11, 18 and 36 catalysts (figures 8 and 9). The degradation rate followed the order AlPO-5 $>$ AlPO-18 $>$ AlPO-11 $>$ AlPO-36 in both the cases of metal doped AlPOs.

Langmuir-Hinshelwood $(\mathrm{L}-\mathrm{H})$ kinetics of the form $r_{0}=k_{0} C_{0} /\left(1+K_{0} C_{0}\right),\left(r_{0}\right.$ is the initial rate, $C_{0}$ is the initial concentration of the dye, $k_{0}$ the kinetic rate constant and $K_{0}$ is the equivalent adsorption coefficient) was employed to quantify the photochemical degradation reaction. The plots of the reciprocal initial degradation rate $\left(1 / r_{\mathrm{o}}\right)$ with the reciprocal of the initial dye concentration $\left(1 / C_{0}\right)$ in presence of MgAlPO-5, 11, 18, 36 and TiAlPO-5, 11, 18, 36 are shown in figures $10 \mathrm{a}$ and $\mathrm{b}$, respectively. The parameters, $k_{0}$ and $K_{0}$, for the photocatalytic degradation of the dyes are obtained from the slope and the intercept of the linear plot (table 1) for the various MeAlPOs. The degradation rate coefficient, $k_{0}$, for $\mathrm{MB}$ in the presence of MgAlPO-5 is more than twice that of MgAlPO-11, 18 and 36. Similarly, the degradation rate coefficient for $\mathrm{MB}$ in presence of TiAlPO-5 is nearly twice that of MgAlPO-5, TiAlPO-11, 18 and 36.

From the above studies, it appears that TiAlPO-5 and $\mathrm{MgAlPO}-5$ are better catalysts and we carried out a detailed study for the photodegradation of different classes of dyes with different initial concentrations. For this, we took 50, 40, 30 and $20 \mathrm{ppm}$ concentration of RBL (xanthene type dye; $\lambda_{\max }=$ $663 \mathrm{~nm}$ ), 100, 75, 50 and $25 \mathrm{ppm}$ of OG (azoic type dye; $\lambda_{\max }=482 \mathrm{~nm}$ ) and DSMP (ionic type dye; $\left.\lambda_{\max }=450 \mathrm{~nm}\right) 100,75,50$ and $30 \mathrm{ppm}$ of AG (anthraquinoic type dye; $\lambda_{\max }=604 \mathrm{~nm}$ ) dye in the presence of $2 \mathrm{~g} / \mathrm{L}$ of MgAlPO-5 (8 atom \%) catalyst (figure 11a). In all the cases, we observed a reasonable degradation of the dyes. When the similar studies were carried out with TiAlPO-5, better 

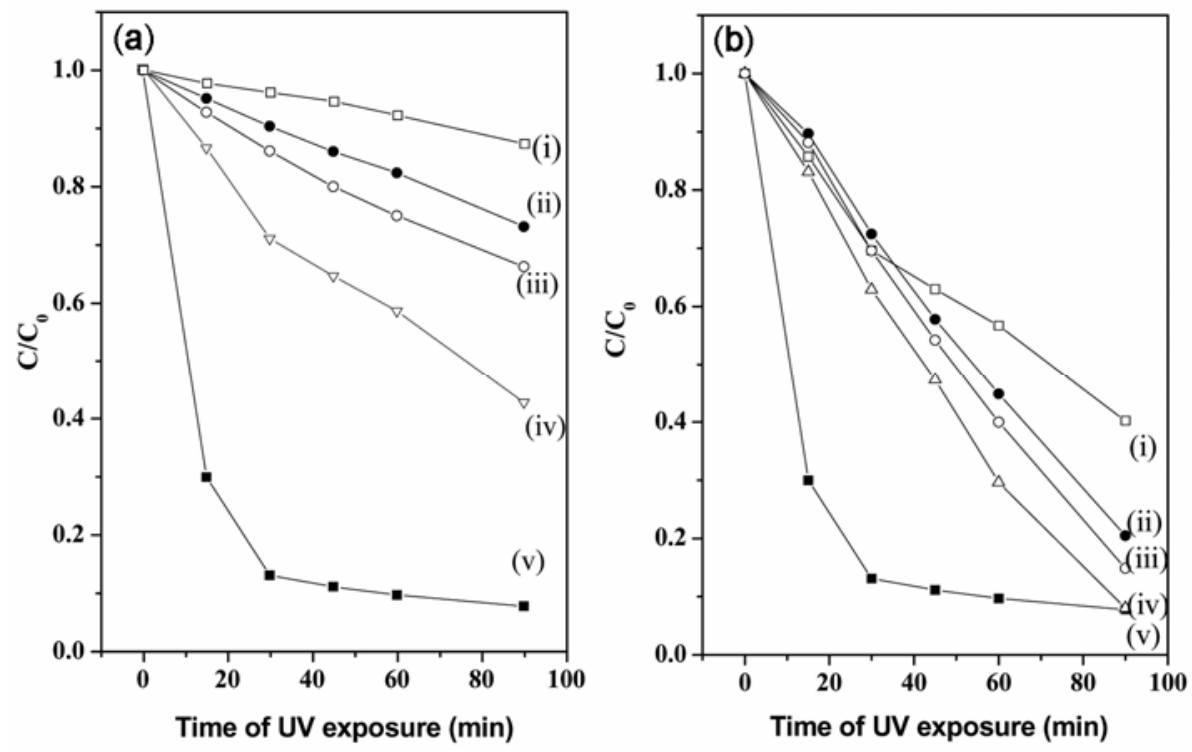

Figure 6. Degradation profiles of MB with an initial concentration of $25 \mathrm{ppm}$ by (a) (i) $4 \%$, (ii) $8 \%$, (iii) $12 \%$ doped MgAlPO-5 (2 g/L), (iv) Degussa P25 $(0.2 \mathrm{~g} / \mathrm{L})$ and (v) Degussa P25 (2 g/L); (b) (i) Degussa P25 (0.2 g/L) (ii) 4\%, (iii) $8 \%$, (iv) $12 \%$ doped TiAlPO-5 (2 g/L) and (v) Degussa P25 (2 g/L).
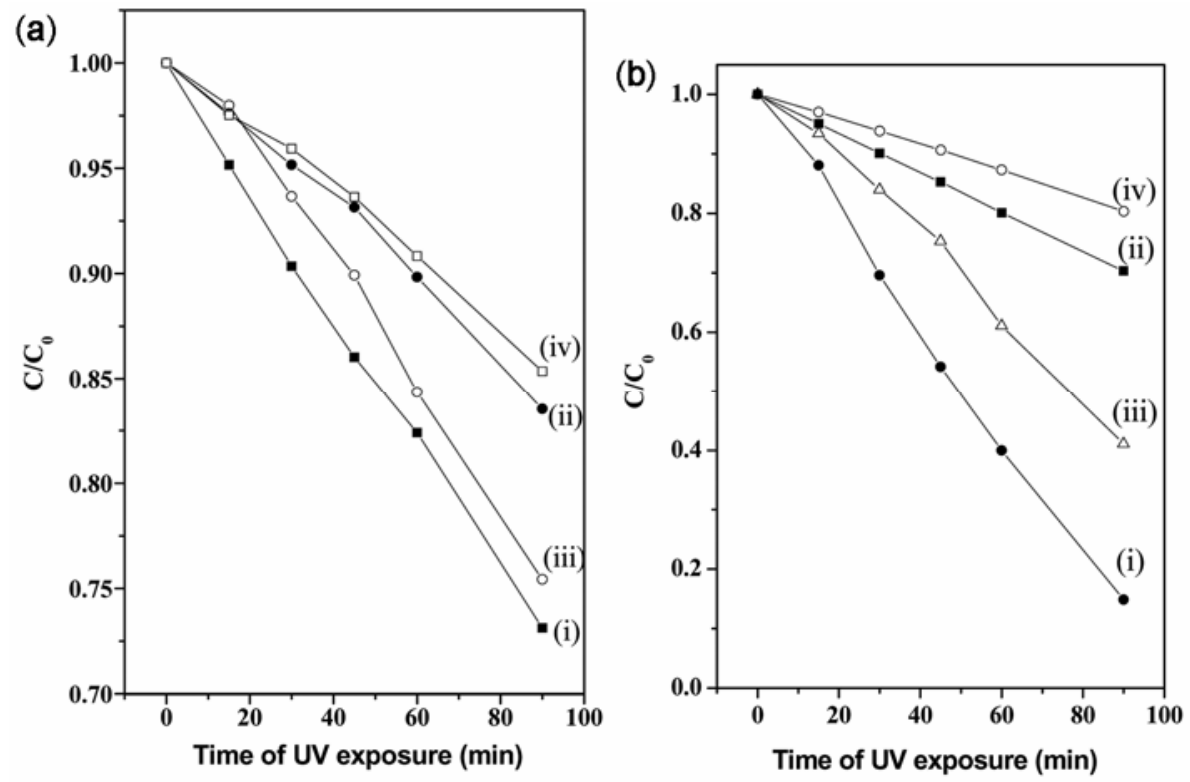

Figure 7. Degradation profiles of MB with an initial concentration of $25 \mathrm{ppm}$ in presence of (a) $8 \% \mathrm{Mg}$ doped (i) AlPO-5, (ii) AlPO-11, (iii) AlPO-18 and (iv) AlPO-36. (b) $8 \%$ Ti doped (i) AlPO-5, (ii) AlPO-11, (iii) AlPO-18 and (iv) AlPO-36.

degradation rates were obtained as compared to the MgAlPO-5 (figure 11b). In all the cases, the variations of the dye concentration as a function of time for different initial concentrations are presented in figures 12 and 13. The rate constants were measured and compared for both the $\mathrm{Mg}$ and the Ti doped 

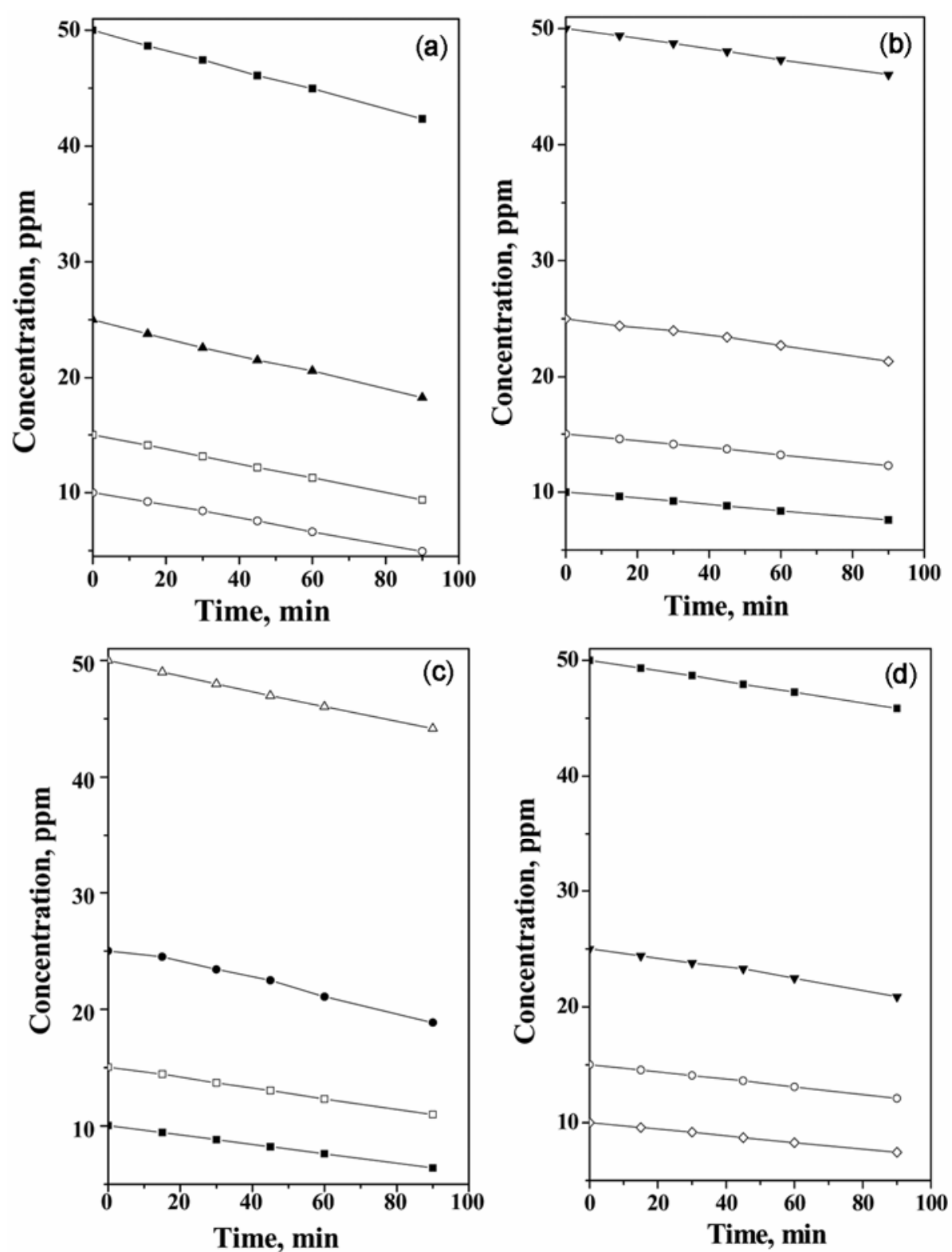

Figure 8. Degradation profile of $M B$ with different initial concentration in presence of (a) MgALPO-11; (b) MgALPO-11; (c) MgALPO-18 and (d) MgALPO-36 respectively.

Table 1. Kinetic parameters for the degradation of $\mathrm{MB}$ dye with $8 \% \mathrm{Mg}^{+2}$ ion doped $8 \% \mathrm{Ti}^{+4}$ ion doped AlPO-5, 11, 18 and 36 .

\begin{tabular}{lcccc}
\hline $\begin{array}{l}\text { Compound } \\
(8 \% \text { doped })\end{array}$ & $\begin{array}{c}\mathrm{Mg}^{+2} \text { doped } \\
k_{0}\left(\mathrm{~min}^{-1}\right)\end{array}$ & $\begin{array}{c}\mathrm{Mg}^{+2} \text { doped } \\
K_{0}\left(\mathrm{ppm}^{-1}\right)\end{array}$ & $\begin{array}{c}\mathrm{Ti}^{+4} \text { doped } \\
k_{0}\left(\mathrm{~min}^{-1}\right)\end{array}$ & $\begin{array}{c}\mathrm{Ti}^{+4} \text { doped } \\
K_{0}\left(\mathrm{ppm}^{-1}\right)\end{array}$ \\
\hline AlPO-5 & 0.018 & 0.003 & 0.027 & 0.061 \\
AlPO-11 & 0.006 & 0.105 & 0.013 & $0 \cdot 100$ \\
AlPO-18 & 0.007 & 0.069 & 0.016 & 0.066 \\
AlPO-36 & 0.005 & 0.098 & 0.010 & $0 \cdot 140$ \\
\hline
\end{tabular}

AlPO-5 (table 2), which consistently showed a higher degradation rate for the TiAlPO-5 compared to MgAlPO-5.
From the above studies, it is clear that the TiAlPO-5 (8 atom \%) and the MgAlPO-5 (8 atom $\%)$ appear to exhibit good activity as a photocatalyst 

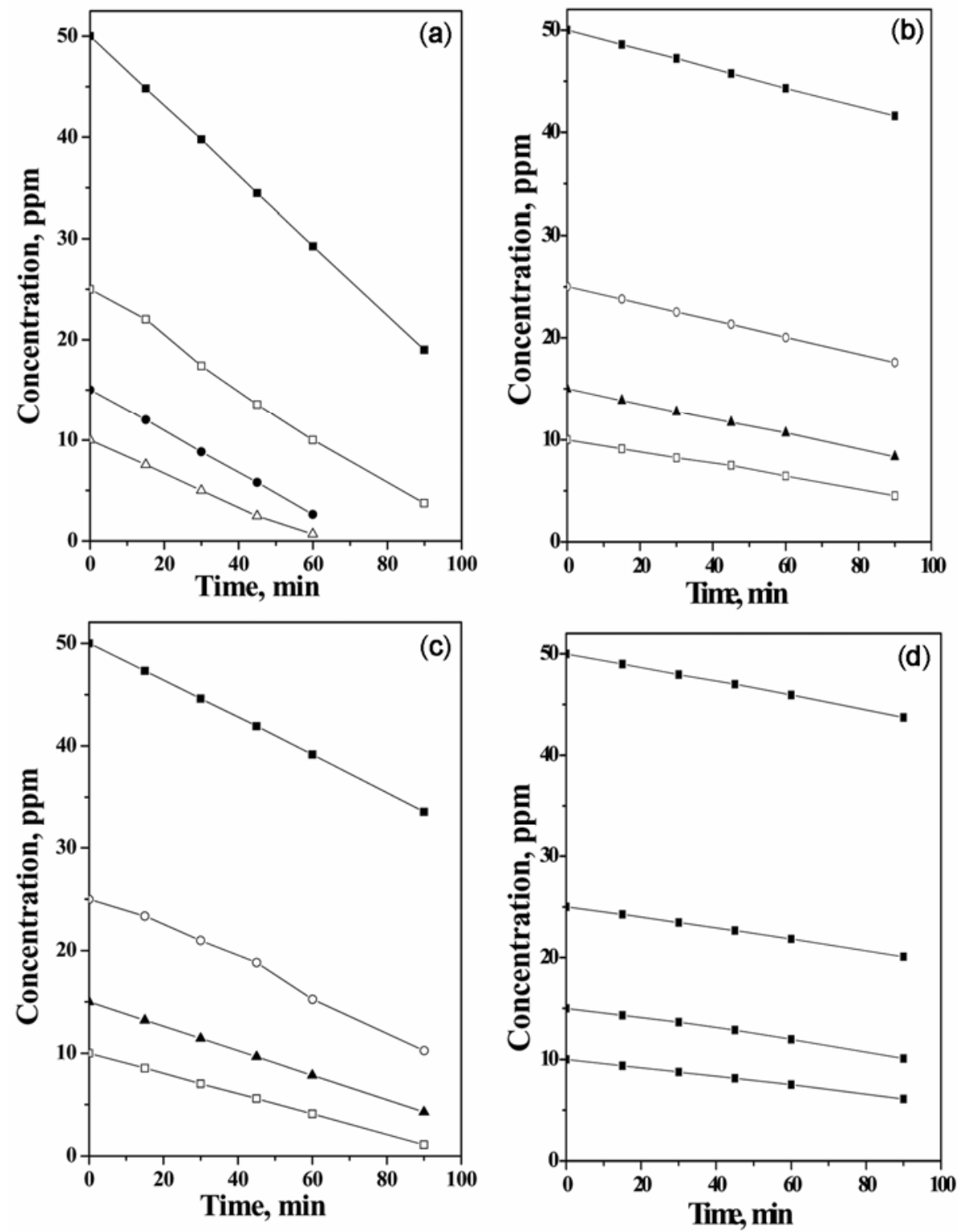

Figure 9. Degradation profile of $\mathrm{MB}$ with different initial concentration in presence of (a) TiAlPO-5; (b) TiALPO-11; (c) TiALPO-18 and (d) TiALPO-36 respectively.

Table 2. Kinetic parameters for the degradation of dyes with $8 \% \mathrm{Mg}^{+2}$ ion doped and $8 \% \mathrm{Ti}^{+4}$ ion doped AlPO-5.

\begin{tabular}{|c|c|c|c|c|}
\hline $\begin{array}{l}\text { Name of the } \\
\text { dye }\end{array}$ & $\begin{array}{l}8 \% \mathrm{Mg}^{+2} \\
k_{0}\left(\min ^{-1}\right)\end{array}$ & $\begin{array}{c}8 \% \mathrm{Mg}^{+2} \\
K_{0}\left(\mathrm{ppm}^{-1}\right)\end{array}$ & $\begin{array}{c}8 \% \mathrm{Ti}^{+4} \\
k_{0}\left(\min ^{-1}\right)\end{array}$ & $\begin{array}{c}8 \% \mathrm{Ti}^{+4} \\
K_{0}\left(\mathrm{ppm}^{-1}\right)\end{array}$ \\
\hline MB & 0.018 & 0.003 & 0.027 & 0.061 \\
\hline RBL & $0 \cdot 017$ & $0 \cdot 048$ & 0.074 & 0.087 \\
\hline $\mathrm{OG}$ & 0.003 & 0.008 & 0.021 & $0 \cdot 038$ \\
\hline $\mathrm{AG}$ & 0.003 & 0.002 & 0.013 & $0 \cdot 012$ \\
\hline DSMP & 0.022 & 0.087 & 0.029 & $0 \cdot 045$ \\
\hline
\end{tabular}

for the degradation of the organic dyes. It may be worthwhile to ponder over the possible reason for this observation.
The FTIR spectroscopic studies indicated an extra peak at $3615 \mathrm{~cm}^{-1}$ for TiAlPO-5, which could be a $-\mathrm{OH}$ group (hydroxyl). The presence of $-\mathrm{OH}$ group 

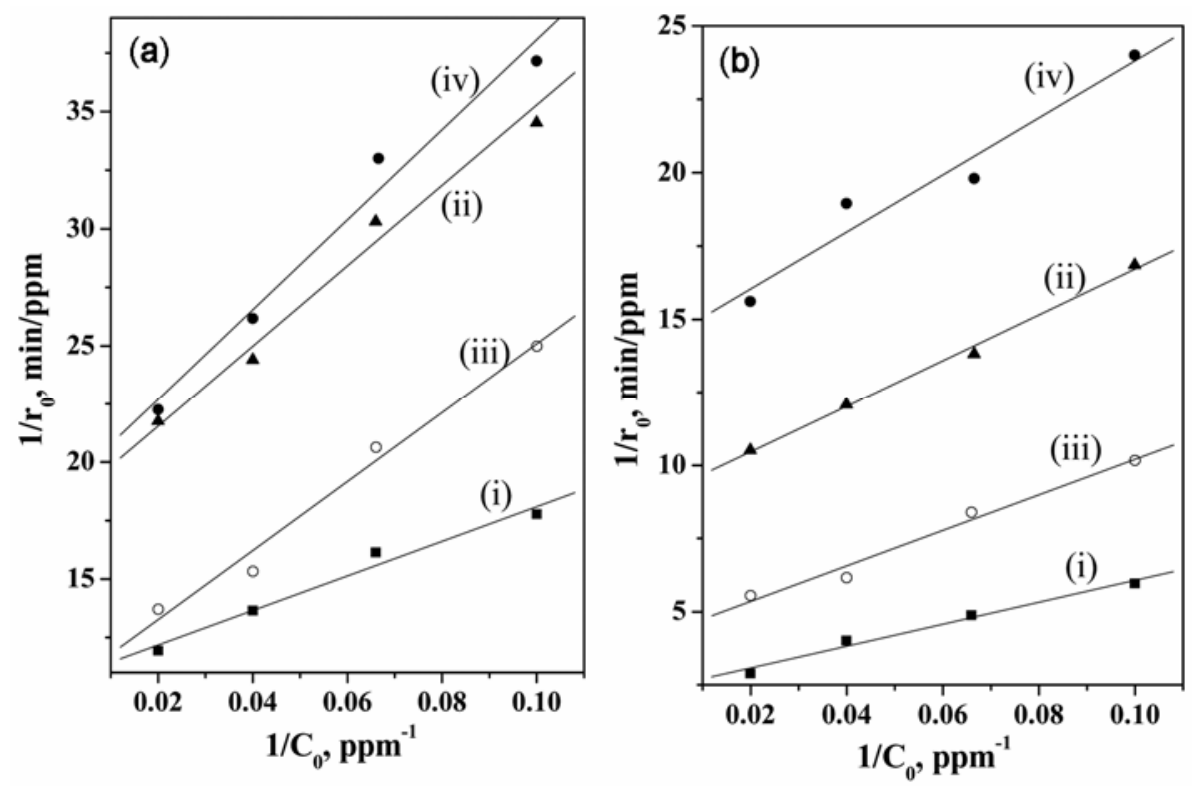

Figure 10. Variation of the photodegradation rate with the initial concentration of $\mathrm{MB}$ in presence of $2 \mathrm{~g} / \mathrm{L}$ of $8 \%$ doped (a) (i) $\mathrm{MgAlPO}-5$, (ii) MgAlPO11, (iii) MgAlPO-18 and (iv) MgAlPO-36. (b) (i) TiAlPO-5, (ii) TiAlPO-11, (iii) TiAlPO-18 and (iv) TiAlPO-36.
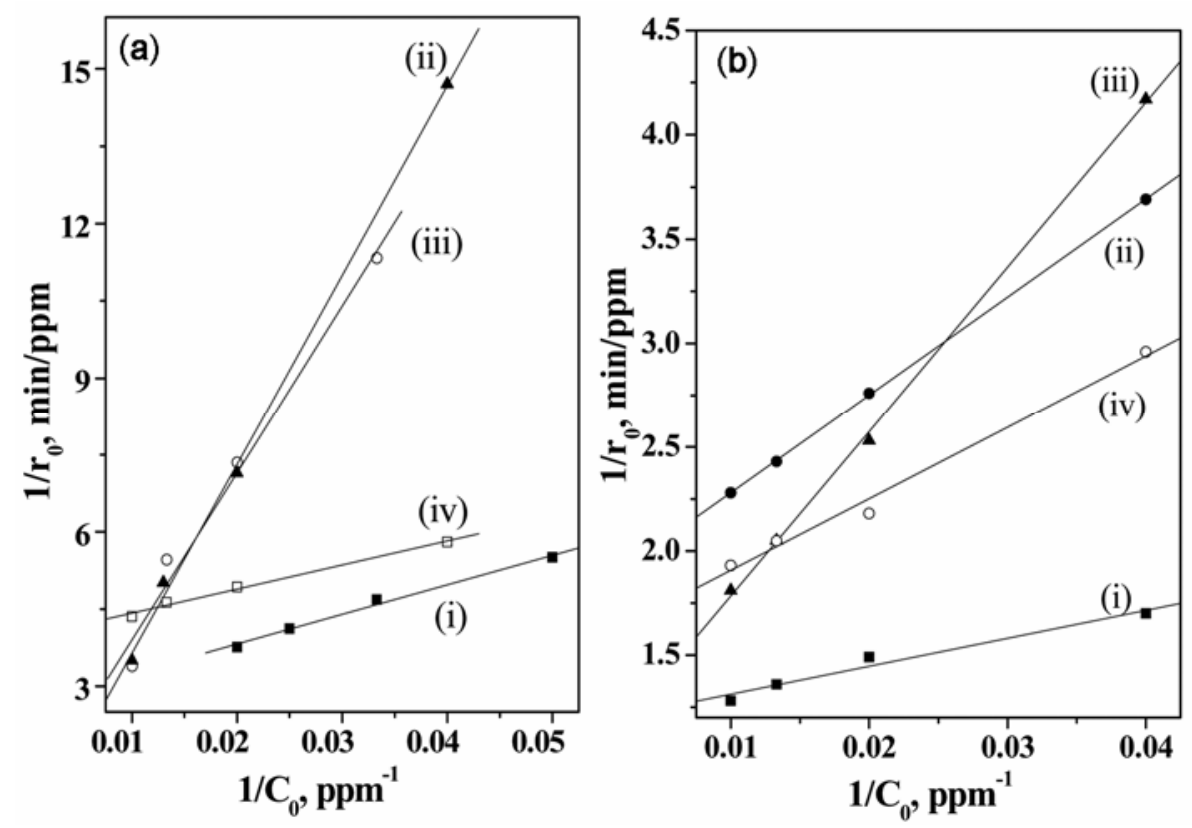

Figure 11. Variation of the photodegradation rate with the initial concentration of (i) RBL (ii) OG (iii) AG and (iv) DSMP in presence of $2 \mathrm{~g} / \mathrm{L}$ of $8 \%$ (a) MgAlPO-5; (b) TiAlPO-5.

can be attributed to the substitution of $\mathrm{Ti}^{+4}$ in place of $\mathrm{Al}^{+3}$ in the framework of AlPO-5. It is likely that the $-\mathrm{OH}$ group is labile, similar to the $\mathrm{H}^{+}$ion in $\mathrm{M}^{+2}$ substituted AlPOs. ${ }^{30}$ The enhanced catalytic activity of the Ti-substituted AlPO-5 may be related to the presence of the $-\mathrm{OH}$ group, though it is a little premature to draw a conclusion. Similarly, the substitution of $\mathrm{Mg}^{+2}$ ion in place of $\mathrm{Al}^{+3}$ can give rise to 

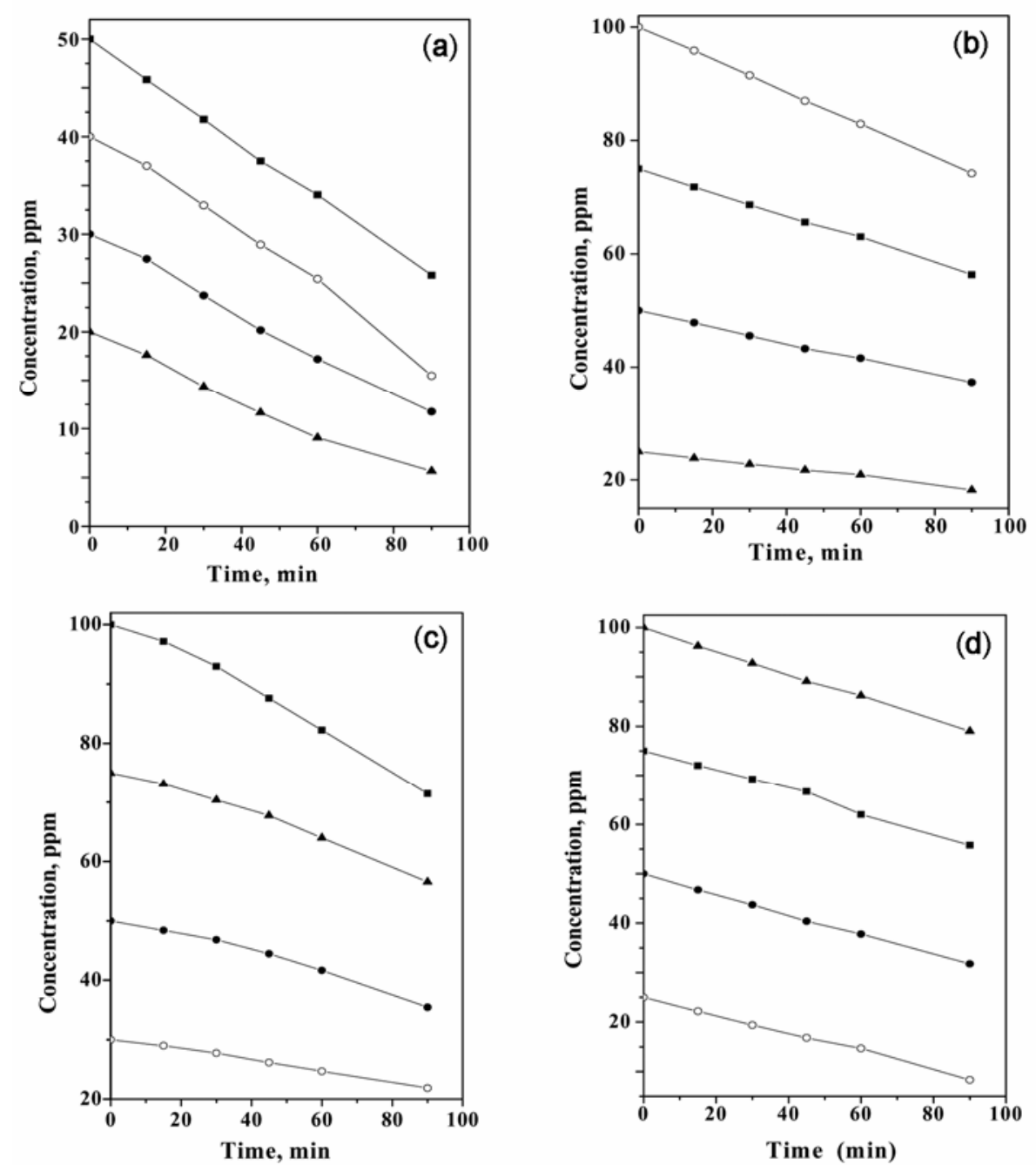

Figure 12. Degradation profile of (a) RBL; (b) OG and (c) AG (d) DSMP with different initial concentration of dyes in the presence of MgALPO-5.

Table 3. Atom \% present in the product AlPO-5 and substituted AlPO-5 obtained from the ICP analysis.

\begin{tabular}{lc}
\hline Compounds (initial atom \%) & Elements present in product (atom \%) \\
\hline AlPO-5 & $\mathrm{Al}=50 \cdot 5, \mathrm{P}=49 \cdot 5$ \\
MgAlPO-5 (4 at\%) & $\mathrm{Mg}=2 \cdot 1, \mathrm{Al}=48 \cdot 2, \mathrm{P}=49 \cdot 7$ \\
MgAlPO-5 $(8 \mathrm{at} \%)$ & $\mathrm{Mg}=4 \cdot 3, \mathrm{Al}=46 \cdot 7, \mathrm{P}=49$ \\
MgAlPO-5 $(12 \mathrm{at} \%)$ & $\mathrm{Mg}=6 \cdot 3, \mathrm{Al}=44 \cdot 4, \mathrm{P}=49 \cdot 3$ \\
TiAlPO-5 $(4 \mathrm{at} \%)$ & $\mathrm{Ti}=2 \cdot 2, \mathrm{Al}=49 \cdot 3, \mathrm{P}=48 \cdot 5$ \\
TiAlPO-5 $(8 \mathrm{at} \%)$ & $\mathrm{Ti}=4, \mathrm{Al}=48, \mathrm{P}=48$ \\
TiAlPO-5 $(12 \mathrm{at} \%)$ & $\mathrm{Ti}=6 \cdot 3, \mathrm{Al}=45 \cdot 7, \mathrm{P}=48$ \\
\hline
\end{tabular}

labile $\mathrm{H}^{+}$ions (resulting in a Bronsted acidity), which could contribute to the catalytic behaviour. The substitution of $\mathrm{Zn}^{+2}$ and $\mathrm{Co}^{2+}$ in place of $\mathrm{Al}^{+3}$ can give rise to $\mathrm{H}^{+}$ions, but the ionic radius of $\mathrm{Zn}^{+2}$ and $\mathrm{Co}^{2+}$ ion $(0.75 \AA)$ is much bigger than of $\mathrm{Al}^{+3}$
$(0.50 \AA)$ which can make the defect sites in original AlPO-5 structure. The original AlPO-5 structure is not distorted by the substitution of smaller ion $\left(\mathrm{Mg}^{+2}, 0.64 \AA\right)$, which is prone to electronic transition from valance bond to conduction band in 

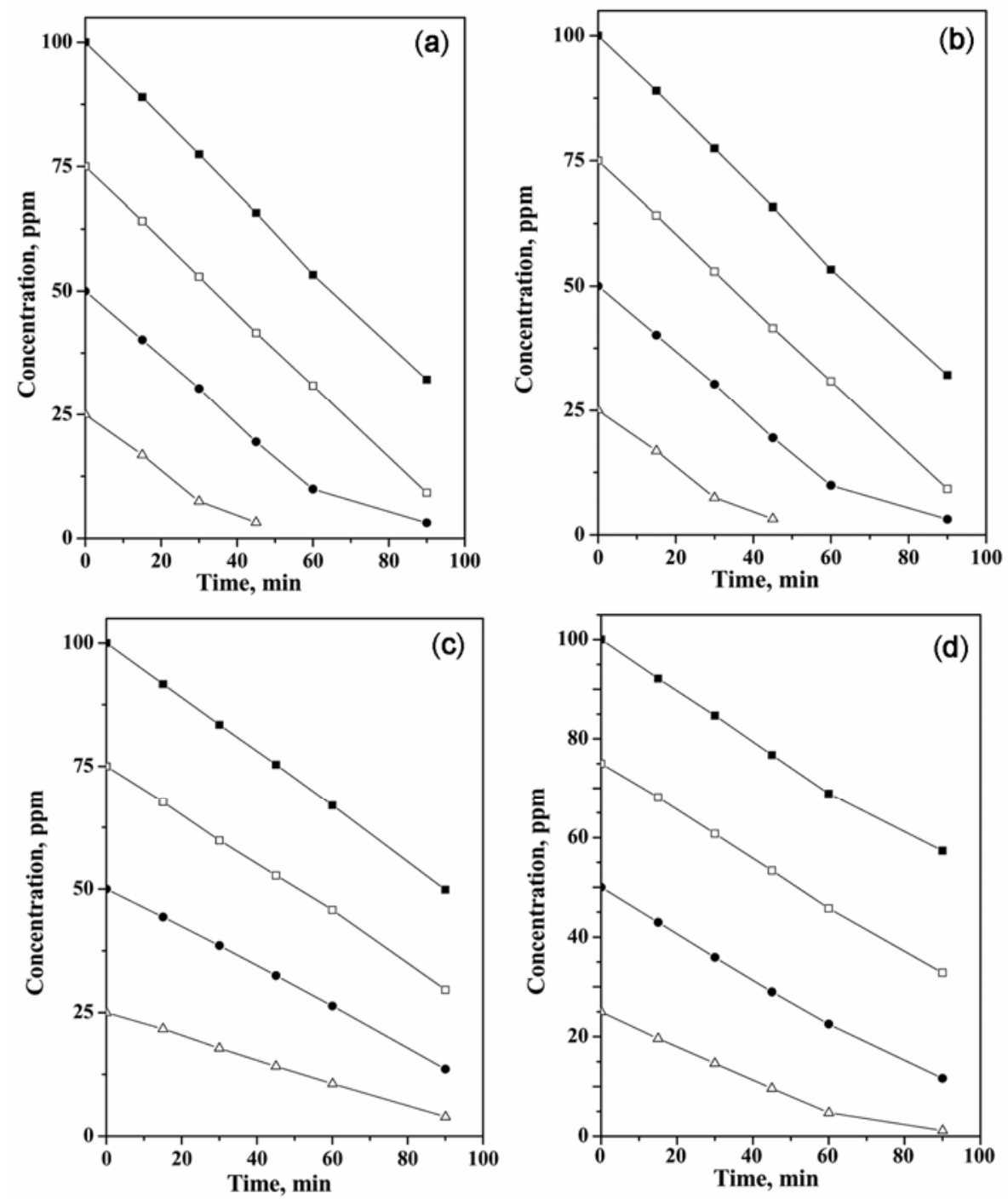

Figure 13. Degradation profile of (a) RBL; (b) OG and (c) AG (d) DSMP with different initial concentration of dyes in the presence of TiALPO-5.

Table 4. Band gap and surface area of AlPOs and substituted AlPOs. All the compounds exhibited a reflectance peak at $\sim 267 \mathrm{~nm}$ corresponding to $4.6 \mathrm{eV}$. The table is for the extra reflectance peak observed including the common band gap of $4.6 \mathrm{eV}$.

\begin{tabular}{lcc}
\hline Compounds & $\begin{array}{c}\text { Band gap } \\
(\mathrm{eV})\end{array}$ & $\begin{array}{c}\text { Surface area } \\
\left(\mathrm{m}^{2} / \mathrm{g}\right)\end{array}$ \\
\hline TiAlPO-5 & $\sim 3.18$ & 224 \\
MgAlPO-5 (4 atom \%) & $\sim 4 \cdot 16$ & 240 \\
MgAlPO-5 (8, 12 atom \%) & $\sim 3.93$ & $230-240$ \\
AlPO-5 & $\sim 4.6$ & 260 \\
CoAlPO-5 & $\sim 4.6$ & 222 \\
ZnAlPO-5 & $\sim 4.6$ & 255 \\
MgAlPO-11 & $\sim 4.6$ & 190 \\
MgAlPO-18 & $\sim 4.6$ & 463 \\
MgAlPO-36 & $\sim 4.6$ & 371 \\
TiAlPO-11 & $\sim 3.18$ & 185 \\
TiAlPO-18 & $\sim 3.18$ & 451 \\
TiAlPO-36 & $\sim 3.18$ & 362 \\
\hline
\end{tabular}

presence of UV light. So, the extra peak in UV-Vis spectra can be found in case of MgAlPO-5. It may be noted that the photocatalytic reactions were carried out in aqueous medium and hence the mechanism of the degradation of the dyes either by $\mathrm{OH}^{-}$or $\mathrm{H}^{+}$ions are likely to be similar and complementary in nature.

To investigate the substitution of $\mathrm{Ti}$ and $\mathrm{Mg}$ in the substituted ALPO-5, the ICP analysis was carried out. The results (table 3) indicate that $\mathrm{Mg}$ replaces $\mathrm{Al}$ but $\mathrm{Ti}$ replaces both $\mathrm{Al}$ and $\mathrm{P}$ in AlPO-5. We have also tried to prepare TiAlPO-5 $\left(\mathrm{Ti}_{\mathrm{X}} \mathrm{AlP}_{(1-x)} \mathrm{O}_{4}\right)$ with 4,8 and 12 atom \% doping of the $\mathrm{P}$ site to verify the position of Ti in AlPO-5. The PXRD (see figure 14) showed that only 4 at $\%$ TiAlPO can be prepared in pure phase with lower peak intensity. 8 atom \% doping produced a new unknown amorphous phase with TiAlPO-5 and 12 atom \% doping 
gave an amorphous unknown phase. This suggests that $\mathrm{Ti}$ is substituted in both the $\mathrm{Al}$ and $\mathrm{P}$ sites and it could not be prepared by taking the initial composition of $\mathrm{Ti}_{x} \mathrm{AlP}_{(1-x)} \mathrm{O}_{4}$.

Regarding charge balance, the simultaneous substitution of $\mathrm{Ti}^{+4}$ in $\mathrm{Al}^{+3}$ as well as $\mathrm{P}^{+5}$ would result in a neutral compound. A small difference in the substitution levels can give rise to the observed behaviour. When $\mathrm{Ti}^{+4}$ was substituted, it is clear that the doping takes place both at the $\mathrm{Al}^{+3}$ as well as $\mathrm{P}^{+5}$ sites in AlPO-5 (from ICP analysis and PXRD). This would suggest a neutral compound, but the differences in the doping at $\mathrm{Al}^{+3}$ and $\mathrm{P}^{+5}$ sites can give rise to either acidic or basic character for TiAlPO-5. It has been known that $\mathrm{Ti}^{+4}$ is preferred at the $\mathrm{P}^{+5}$ sites, which would give acidic behaviour, similar to the doping of $\mathrm{Mg}^{+2}$ at the $\mathrm{Al}^{+3}$ site. Our IR studies (see figure 2) also indicate the presence of $-\mathrm{OH}$ in the TiAlPO-5, suggesting that the trend is consistent as outlined above. Since the total concentration of $\mathrm{Ti}^{+4}$ is small, it was accommodated in the tetrahedral site. This is also confirmed by the XAFS studies (see figure 15). To verify the state of Ti, XAFS spectra were taken with Ti-HMS (tetrahedral coordination of $\mathrm{Ti}$ ) as the reference. The spectra showed that $\mathrm{Ti}$ is in the tetrahedral coordination.

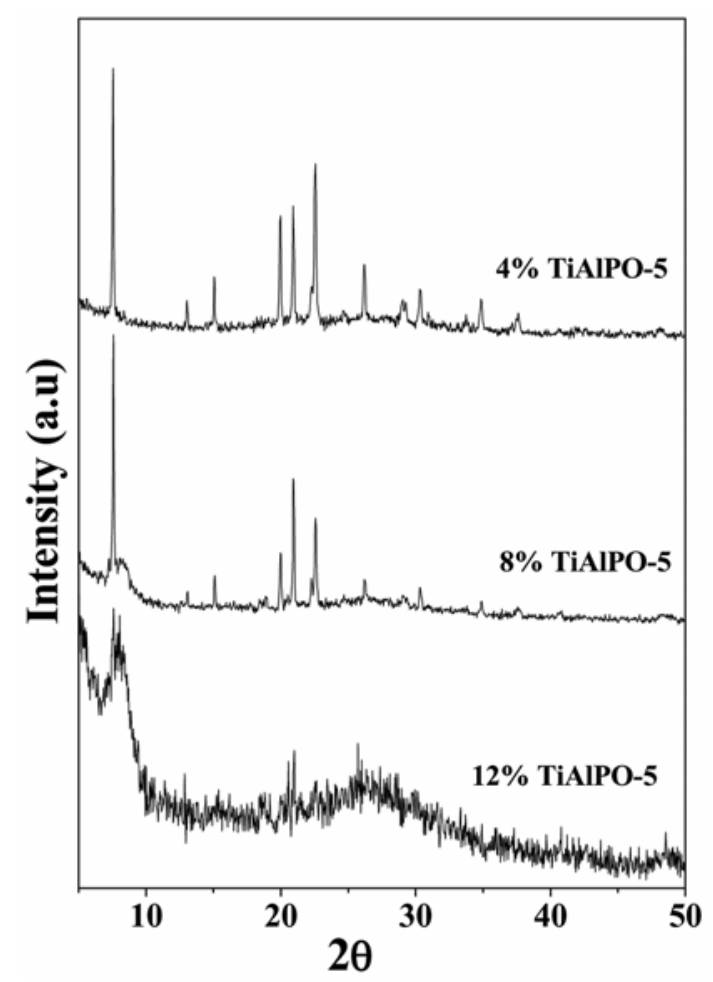

Figure 14. Powder X-ray diffraction pattern $(\mathrm{CuK} \alpha)$ for $\mathrm{Ti}_{\mathrm{X}} \mathrm{AlP}_{(1-x)} \mathrm{O}-5$; where $x$ is 4,8 and 12 at $\%$ doping in place of $\mathrm{P}$ site.
Our diffuse reflectance spectroscopy studies also revealed another interesting feature (figure $3 a$ ) in the $\mathrm{Mg}$ and Ti substituted AlPO-5 samples. In both the cases, the peak corresponding to a band gap of $\sim 3.18$ and $3.93 \mathrm{eV}$ has been observed for the $\mathrm{Ti}$ and Mg- AlPO-5 samples, respectively. It is likely that this band gap could be due to the presence of some defect sites in the original structure. In this context, it is to be noted that the $\mathrm{Co}$ and $\mathrm{Zn}$ substituted AlPO-5 and Mg substituted AlPO-11, 18 and 36 samples do not show any additional features in their diffuse reflectance spectra. The surface areas after substitution of different metal ions in AlPOs were determined and reported in table 4, which are comparable to those reported earlier. ${ }^{31,32}$

The diffuse reflectance spectra for the Ti substituted other AlPOs, viz AlPO-11, 18 and 36 also exhibited similar band corresponding to a band gap of $\sim 3.18 \mathrm{eV}$, but their catalytic activities were found to be lower compared to TiAlPO-5. This observation can be rationalized by comparing the photoluminescence (PL) spectra for all the compounds. Thus, PL

\section{Ti-HMS}

(Ti species: tetrahedral coordination)
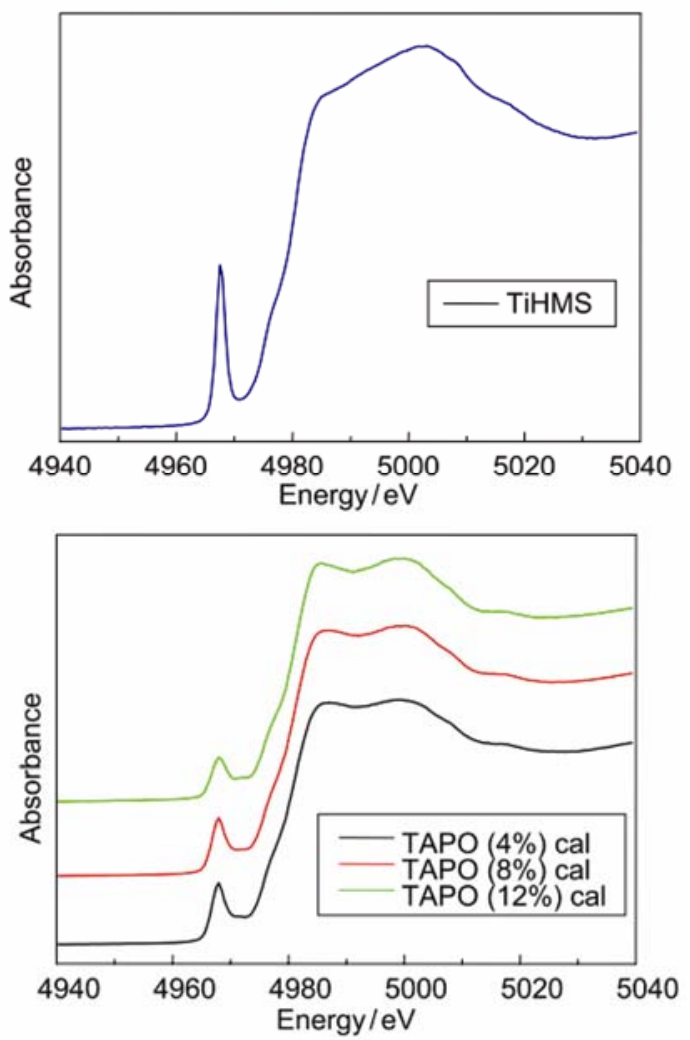

Figure 15. XAFS of Ti-HMS and TiAlPO-5 $(4,8$ and 12 at $\%$ doped). 

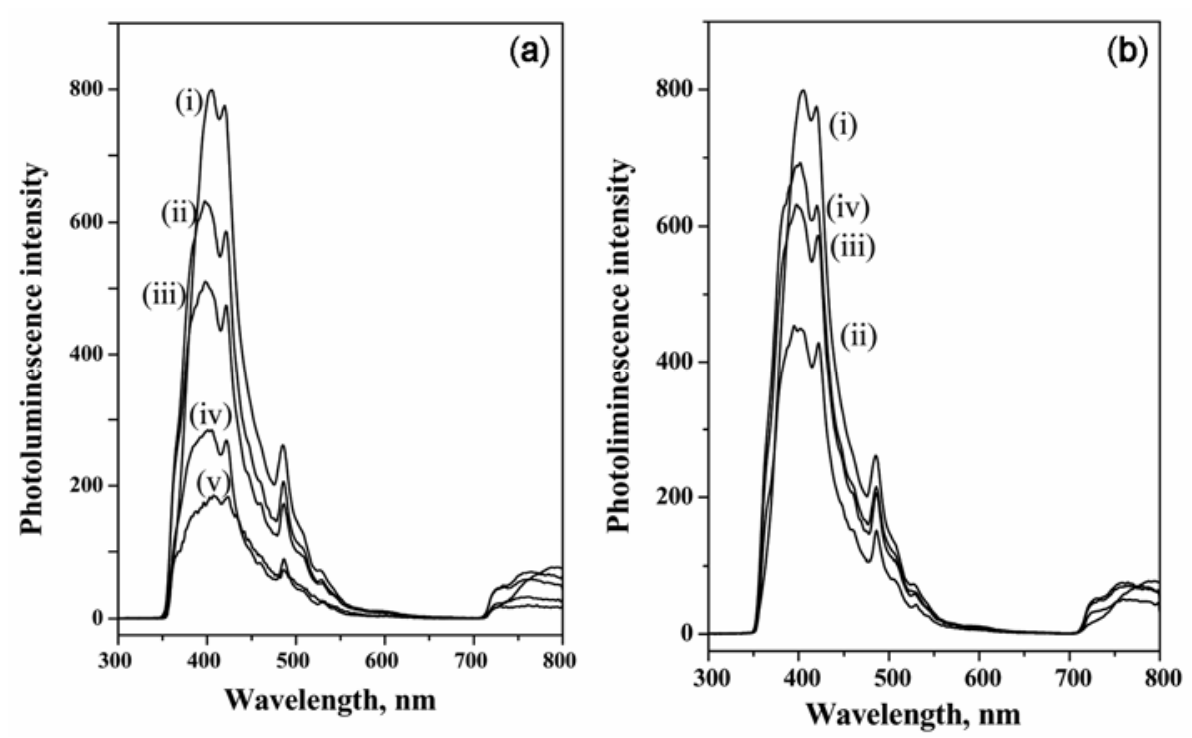

Figure 16. Photoluminescence spectra of (a) (i) $\mathrm{TiO}_{2}$ and $8 \%$ Ti doped; (ii) AlPO-5; (iii) AlPO-18; (iv) AlPO-11 and (v) AlPO-36. (b) (i) $\mathrm{TiO}_{2}$ and (ii) $4 \%$, (iii) $8 \%$, (iv) $12 \%$ doped TiAlPO-5.

spectroscopic studies were carried out using an excitation wavelength of $260 \mathrm{~nm}$ and the results were compared with Degussa P25 $\left(\mathrm{TiO}_{2}\right)$ catalyst. The PL studies have been used as a probe for understanding the primary excitation processes in many semiconductor photocatalysts. ${ }^{33}$ The results of the present study clearly show that the intensity of the emission band at $\sim 484 \mathrm{~nm}$ and the bands at 405 and $419 \mathrm{~nm}$ are the highest for the $\mathrm{TiO}_{2}$ catalyst and reduces consistently for the Ti-doped AlPOs (figure 16a). The band at $484 \mathrm{~nm}$ corresponds to the emission from the $\mathrm{Ti}^{+4}-\mathrm{OH}$ group, ${ }^{33}$ while the bands at 405 and 419 are due to the exciton emission from excited state. ${ }^{34}$ In addition, the PL intensity also increases with the increasing dopant concentration of $\mathrm{Ti}$ in AlPO-5 (figure 16b). Thus, the difference in the photocatalytic activity between the AlPOs could be due to the electron transfer between the excited and the ground state levels of $\mathrm{Ti}$ and follows the PL intensity. The comparison between the data in figures $6 \mathrm{~b}$ and $16 \mathrm{~b}$ clearly shows that the photocatalytic activity increases with increasing PL intensity for different atom \% doping of Ti in AlPO-5. Similarly, Figures $7 \mathrm{~b}$ and $16 \mathrm{a}$ show that the photocatalytic activity increases with increasing PL intensity of Ti doped AlPO- $n(n=5,11,18$ and 36).

\section{Conclusions}

The photocatalytic studies of the metal substituted AlPOs for the degradation of a variety of organic dyes have been investigated and compared to that of Degussa P25 catalyst. The present study suggests that the metal doped aluminophosphates could be employed as a photocatalyst for degradation of organic dyes. Among the AlPOs, AlPO-5 structure seems to be better for photocatalytic activity. Among the metal doped AlPOs, only $\mathrm{Mg}$ and $\mathrm{Ti}$ substituted AlPOs show significant photocatalytic activity. The photocatalytic activity can be enhanced by the increasing of doped metal ion concentration. The degradation rate coefficients of the dyes in presence of Ti doped AlPOs are higher than that of $\mathrm{Mg}$ doped AlPOs. The photocatalytic activity of the Ti-substituted AlPOs has been reasoned with the PL studies.

\section{Supplementary information}

Powder XRD pattern of all MeAlPOs in as prepared form and calcined form by comparing with simulated pattern (figures S1-S10 can be seen in the website as supplementary information. See www.ias. ac.in/chemsci).

\section{Acknowledgments}

G M thanks the Department of Science and Technology (DST), Government of India, for the award of a research grant. S N thanks DST, Government of India, for the award of an Indo-Japan project. A K P 
thanks the Council of Scientific and Industrial Research (CSIR), Government of India, for the SRF fellowships.

\section{References}

1. Thomas J M 1992 Sci. Am. 266112

2. Barrer R M 1989 Pure Appl. Chem. 611903

3. Vaughan D E W 1989 in Zeolite, facts, figures and future (eds) P A Jacobs and R A (Van Santen: Elsevier)

4. Thomas J M and Thomas W J 1997 Heterogeneous catalysis (Wiley-VCG)

5. Wilson S T, Lok B M, Messina C A, Cannan T R and Flanigen E M 1982 J. Am. Chem. Soc. 1041146

6. Lok B M, Messina C A, Patton R L, Gajek R T, Cannan T R and Flanigen E M $1984 \mathrm{~J}$. Am. Chem. Soc. 1066092

7. Thomas J M and Raja R 2001 Chem. Rec. 1 448

8. Raja R, Thomas J M, Greenhill-Hooper $\mathrm{M}$ and Doukova V 2007 Chem. Commun. 1924

9. Ramamurthy V and Schanze K (eds) 2006 Molecular and supramolecular photochemistry. Organic photochemistry and photophysics (Taylor and Francis: Boca Raton) Vol. 14

10. Natarajan A, Kaanumalle L S, Jockusch S, Gibb C L D, Gibb B C, Turro N J and Ramamurthy V 2007 J. Am. Chem. Soc. 1294132

11. $\mathrm{Hu} Y$, Rakhmawaty D, Matsuoka M, Takeuchi M and Anpo M 2006 J. Porous. Mater. 13335

12. Hoffmann M R, Martin S T, Choi W and Bahnemann D W 1995 Chem. Rev. 9569

13. Linsebrigler A L, Lu G and Yates J T 1995 Chem. Rev. 95735

14. Wang $\mathrm{P}$, Huang B, Qin X, Zhang X, Dai Y, Wei J and Whangbo M-H 2008 Angew. Chem. Int. Ed. 47 7931
15. Wei W, Dai Y and Huang B 2009 J. Phys. Chem. C113 5658

16. Cheng H, Huang B, Dai Y, Qin X, Zhang X, Wang Z and Jiang M 2009 J. Solid State Chem. 1822274

17. Anpo M and Thomas J M 2006 Chem. Commun. 3273

18. Ranjit K T, Uma S, Martyanov I N and Klabunde $\mathrm{K} \mathrm{J}$ 2005 Adv. Mater. 172467

19. Sivalingam G, Nagaveni K, Hegde M S and Madras G 2003 Appl. Catal. B45 23

20. Mahapatra S, Madras G and Guru Row T N 2007 J. Phys. Chem. C111 6505

21. Mahata P, Aarthi T, Madras G and Natarajan S 2007 J. Phys. Chem. C111 1665

22. Hislop K A and Bolton J R 1999 Environ. Sci. Technol. 333119

23. Mahata P, Madras G and Natarajan S 2006 J. Phys. Chem. B110 13759

24. Mahata P, Sankar G, Madras G and Natarajan S 2005 Chem. Commun. 5787

25. Mahata P, Madras G and Natarajan S 2007 Catal. Lett. 11527

26. Xiulam W, Yang Y G and Hanfu L 2005 Molecular Catalysis China 19477

27. http://www.iza-structure.org/databases/

28. Paul M, Pal N, Rana B S, Sinha A K and Bhaumik A 2009 Catal. Commun. 102041

29. Zahedi-Naiki M H, Kapoor M P and Kaliaguine S 1998 J. Catal. 177231

30. Smith L, Cheetham A K, Morris R E, Marchese L, Thomas J M and Wright P A 1996 J. Chen. Sci. 271 799

31. Chen C-M and Jehng J-M 2004 Catal. Lett. 93213

32. Zahedi-Niaki M H, Joshi P N and Kaliaguine S 1996 Chem. Commun. 47

33. Nagaveni K, Hegde M S and Madras G 2004 J. Phys. Chem. B108 20204

34. Vinu R and Madras G $2008 \mathrm{~J}$. Mol. Catal A: Chem. 2915 